\title{
Obtaining the Distribution of Quiescent Periods Directly from the Power Spectral Densities of Sea Waves
}

Michael Belmont*, Mustafa Al-Ani, Peter Challenor and Jacqueline Christmas

College of Engineering, Mathematics and Physical Sciences, University of Exeter, Exeter, UK.

Philip Wilson

Faculty of Engineering and Physical Sciences, University of Southampton, Southampton, UK.

* Corresponding author. E-mail address: m.r.belmont@exeter.ac.uk (M. R. Belmont) 


\section{ABSTRACT}

There is a growing practical interest in the ability to increase the sea states at which marine operations can be safely undertaken by exploiting the quiescent periods that are well known to exist under a wide range of sea conditions. While the actual prediction of quiescent periods at sea for the control of operations is a deterministic process, the long term planning of future maritime tasks that rely on these quiescent periods is a statistical process involving the anticipated quiescence properties of the forecasted sea conditions in the geographical region of interest. It is in principle possible to obtain such data in tabular form either large scale simulation or from field data. However, such simulations are computationally intensive and libraries of appropriate field data are not common. Thus, it is clearly attractive to develop techniques that exploit standard wave spectral models for describing the quiescence statistics directly from such spectra. The present study focuses upon such techniques and is a first step towards the production of a computationally low-cost quiescence prediction tool and compares its efficacy against simulations. Two significant properties emerge for a large class of wave spectral models that encompasses the ubiquitous Neumann and Pierson Moskowitz or Bretschneider forms. Firstly, the auto-correlation function of the wave profile that are required to produce the quiescence property can be obtained analytically in terms of standard special functions. This considerably reduces the computational cost making desktop computer-based planning tools a reality. Secondly, for each class of these parametric spectra, the probability of a given number of consecutive wave heights (normalised to the significant wave heights) less than some critical value is in fact independent of absolute wave height. Thus, for a broad class of practically interesting wave spectra all that is required to obtain the statistical distribution of the quiescent periods is simple rescaling.

Keywords - Deterministic sea wave prediction (DSWP), quiescent period prediction (QPP), wave runs.

\section{Introduction}

There is a rapidly growing applications interest [1] - [3] in the ability to exploit the successions of small waves, termed quiescent periods (QPs) that are well known to exist in otherwise large seas. The ability to deterministically predict such QPs even a few tens of seconds into the future offers the prospect of allowing a marked increase in the sea states under which a variety of maritime operations can be safely performed. As the short-term deterministic prediction of sea waves and its applications is a new field in applied oceanography it is sensible to briefly describe the basis of the technique and to provide illustrative applications. This is especially important because the time-scales of interest are tens of seconds which would appear as trivially short and of no practical value in traditional oceanographic terms.

The underpinning new discipline behind quiescent period prediction (QPP) is termed Deterministic Sea Wave Prediction (DSWP) [4] - [7]. This should be distinguished from the more conventional statistical description of sea waves. DSWP involves firstly measuring the sea surface profile over a region around the site of interest out to a few km distant, typically using the new generation of wave profiling radars [6,7]. The resulting two dimensional wave data sets are then used as the initial condition for a wave

propagation model. The predict-ahead time available is given by the time taken for the measured waves to 
propagate from the measured location to the prediction site, typically 30 to 120 seconds. Given that all the calculations involved must be completed in times short compared to this predict ahead period, the wave models used in DSWP are usually linear. Non-linear DSWP [8] is only viable in real time for highly one dimensional seas. As discussed in the DSWP literature, e.g., [4,5], this linear constraint is less restrictive than might appear and allows useful exploitation of the technique. The main aim of DSWP is in predicting the quiescent periods, i.e., runs of successive waves less than some critical height. Such QPs can be thought of as a generalisation of the phenomenon of beats; and for moderately narrow-band phenomena like fully developed sea waves, the duration of these periods is approximately inversely proportional to the bandwidth of the wave spectrum. This is a useful property because for the reasonably well developed seas that are of most relevance in DSWP based applications the bandwidth reduces with increasing size of the wave system, so larger seas where QPP is of most value exhibit increasingly longer QPs.

The short prediction horizon of DSWP, and hence QPP, is useful because there are many lengthy marine operations where the sub-task which sets the acceptable limiting wave height is actually of very short duration, often prolonging this sub-task rapidly increases risk. Many such applications involve choosing the best time to initiate this wave-height-critical phase of the overall operation. Examples are: cargo hoists from a vessel to a fixed offshore platform (when to start hoisting), recovery of a helicopter (when to land from the over-deck hover), the final critical stages of recovery of remotely operated small submersibles vehicles (when to hoist over the vessel side) and the final connect and lift stages of recovering a small surface craft onto a mother-ship. Most of these tasks involve either stationary vessels under dynamic positioning control or vessel moving at low speed. This means that encounter frequency effects are relatively insignificant and can typically be ignored, the main significance of ship motion being changes in the location of the prediction site during the propagation duration of the waves from where they are measured to the prediction site.

Thus, for QPP to be of practical value, two aspects are required. The first is knowledge of the statistical properties of QPs, i.e. knowledge of the probability of QPs under the anticipated or prevailing sea conditions. This is a vital operational forward planning requirement because clearly one cannot rely on exploiting QPs if they have a very low probability of occurrence and have durations that are too short to be useful. The second aspect is the deterministic side of QPs, i.e. the DSWP described above, which provides the ability to deterministically predict at sea in real time when they will actually occur and what their durations are.

Any vessel wishing to exploit QPP will of necessity be equipped with vessel motion sensors and clearly just prior to executing a mission it is a simple matter to continuously measure the statistics of QPs of the vessel (which is the actual operational requirement rather than merely the QPs of the wave system). However, to produce reliable estimates, a long observation window is required especially for the low probability events of long QPs. Additionally, a major benefit stemming from the availability of QPs information is its use in future mission planning. Consequently it would be highly desirable to be able to describe QPs statistics far in advance of the proposed maritime operation for the typical sea characteristics for the regions and seasons of interest. 
Apart from the direct empirical determination of the statistical distribution of QPs at sea at the time of interest there are three further possible approaches to obtain the statistics of QPs. The first is a semianalytical technique where mathematical models of the power spectral density of the sea type are employed in conjunction with analytic statistical models of the bivariate successive wave heights probability distributions. In this case somewhat surprisingly the bulk of the computations can be accomplished analytically producing a computationally cheap approach. The analysis employed in developing this semi-analytic approach will be shown to reveal that for a wide class of wave spectra the probability distribution of QPs is independent of wave absolute wave height. This property enables further reduction in the computational costs associated with the technique. The second approach to determining the probabilities of QPs is to use purely empirical models of the wave power spectral densities. In this case all of the necessary computations are performed numerically. This method is clearly broader in the scope of sea conditions it can deal with but it is more computationally intense than the semi-analytic method. The third methodology is to collect data from a large scale sea simulation based numerical experiment. This is even more computational intensive than the second methodology described above. The present report deals with the semi-analytic approach which also provides the mathematical basis for the second (numerically evaluated) approach. The latter methodology is the subject of a companion communication.

It transpires that the general background theory required has been extensively covered in what are in the main rather mature publications dating from the 1960's to the 1980's dealing with statistical wave theory. However, the predominant practical motivation which drove such research was in the complementary problem, i.e., sequences of consecutive large rather than small waves. The present treatment will explore the most appropriate results from this literature, modifying and extending these where necessary, and applying the results obtained to the problem of producing the properties of QPs based upon typical spectral wave models.

\section{Runs Theory for Sea Waves}

In statistical terms a run is a sequence of outcomes that all share some property, in this case waves either greater than or less than some critical value $h_{c}$. For entirely obvious engineering reasons the majority of the previous research interest has been focussed on sequences of consecutive large waves which are typically termed, High Runs. The simplest approach to modelling runs of sea waves was developed by Goda [9] who assumed that consecutive waves are statistically independent. As alluded to above Goda's work concentrated upon what at the time was the most important case i.e., High Runs. However using Goda's formalism a Low Run, of length $j$ can be represented in the following manner: the run commences with a first wave having an height less than $h_{c}$, which has a probability of $p\left(h_{c}\right)$, and ends with a wave of height more than $h_{c}$, which has a probability of $1-p\left(h_{c}\right)$. Thus, the probability $P_{j}\left(h_{c}\right)$ of such a low run, which is $j$ waves long, is simply given by:

$$
P_{j}\left(h_{c}\right)=p^{j}\left(h_{c}\right)\left[1-p\left(h_{c}\right)\right]
$$

Equation (1) is evaluated using the standard Rayleigh marginal probability distribution for wave heights. Goda's simple approach ignored the short term correlations that are well known to typically exist over up 
to three "half waves" ahead [10] - [12]. This prompted two more sophisticated approaches in the literature to describe the runs statistics of sea waves. One class of technique termed Wave Envelope methods was based directly upon Rice's work [13,14], on narrow band random signals. This area was reviewed in [15] and is essentially a signal processing technique that endeavours to model the properties of narrow band stochastic processes in terms of generalised amplitude modulation (i.e., envelopes). It relies on the simple property that any dynamical system whose frequency response function is localised around the frequency $f_{0}$ with a spread $\delta f_{0}$ where, $\delta f_{0} / f_{0} \ll 1$, is capable of exhibiting oscillatory behaviour at frequencies close to, $f_{0}$, which is modulated by an envelope that fluctuates over timescales of the order of $1 / \delta f_{0}$. This envelope reflects the statistical dependence that affects runs. For sea waves the bandwidth is measured by the parameter $v=\sqrt{\left(m_{0} m_{2} / m_{1}^{2}\right)-1}$, where the $m_{i}$ is the $i^{\text {th }}$ moment of the power density spectrum. Such envelope behaviours are encapsulated in the theory of Analytic Signals, e.g., [16] which uses the Hilbert Transform in the determination of the envelope functions of interest and has been explored in sea wave applications by Longeut-Higgins [15].

The second alternative to Goda's approach, developed by Kimura [17], employs a Markov Chain model which incorporated the appropriate conditional probability functions that depended upon the correlation between successive wave heights. Using simulated wave data, Kimura was able to demonstrate that incorporating such successive wave correlations significantly improved the ability to model runs as compared to [9]. As expected the findings agreed with those of Goda [9] in the uncorrelated limit. Validation using actual sea data was subsequently provided by Battjes [18] who tested Kimura's results using North Sea wave buoy data rather than simulations.

Both Longuet-Higgins [15] and Kimura [17] used the probability distribution developed by Rice $[13,14]$ and hence the narrow band restrictions apply to both methods. The issue of the legitimacy of employing the narrow band approximation is discuss subsequently with the points made by Arhan [19] in this respect being particularly pertinent.

As stated, Kimura [17] (and Longuet-Higgins [15]) used Rice's [13,14] bivariate Rayleigh probability density function, $p\left(h_{2}, h_{1}\right)$, for consecutive wave height parameters, $h_{1}$, and, $h_{2}$ which in Kimura's formalism is given by:

$$
p\left(h_{2}, h_{1}\right)=\frac{h_{1} h_{2}}{\left(1-\kappa^{2}\right) h_{r m s}^{4}} \exp \left\{\frac{-1}{\left(1-\kappa^{2}\right)} \frac{\left(h_{1}^{2}+h_{2}^{2}\right)}{2 h_{r m s}^{2}}\right\} I_{0}\left\{\frac{h_{1} h_{2} \kappa}{\left(1-\kappa^{2}\right) h_{r m s}^{2}}\right\}
$$

The parameter $I_{0}$ is the modified Bessel function of order zero and the parameter written $\rho$ in Kimura's original treatment is replaced by the more conventional $\kappa$ wave-wave correlation which is equal to $2 \rho$. The phrase, "wave height parameters" for , $h_{1}$, and, $h_{2}$, does not mean surface elevation but as LonguetHiggins [15] points out in Kimura's [17] analysis,$h_{1}$, and, $h_{2}$, are trough to crest values. 
The conditional probabilities required for estimating the probability of a run of low waves that constitutes a quiescent period can be obtained from (2). The family of marginal and conditional probabilities that are relevant are given by:

$$
\begin{gathered}
p_{1}=P\left(h<h_{c}\right)=\int_{0}^{\infty} \int_{0}^{h_{c}} f\left(h_{1}, h_{2}\right) d h_{1} d h_{2} \\
P\left(h>h_{c}\right)=1-p_{1} \\
p_{1,1}=P\left(h_{2}<h_{c} \mid h_{1}<h_{c}\right)=\int_{0}^{h_{c}} \int_{0}^{h_{c}} f\left(h_{1}, h_{2}\right) d h_{1} d h_{2} / p_{L}, \\
p_{2,1}=P\left(h_{2}<h_{c} \mid h_{1}>h_{c}\right)=\int_{0}^{h_{c}} \int_{h_{c}}^{\infty} f\left(h_{1}, h_{2}\right) d h_{1} d h_{2} /\left(1-p_{L}\right), \\
p_{1,2}=P\left(h_{2}>h_{c} \mid h_{1}<h_{c}\right)=\int_{h_{c}}^{\infty} \int_{0}^{h_{c}} f\left(h_{1}, h_{2}\right) d h_{1} d h_{2} / p_{L}, \\
p_{2,2}=P\left(h_{2}>h_{c} \mid h_{1}<h_{c}\right)=\int_{h_{c}}^{\infty} \int_{h_{c}}^{\infty} f\left(h_{1}, h_{2}\right) d h_{1} d h_{2} /\left(1-p_{L}\right),
\end{gathered}
$$


be treated as statistically independent producing an expression for the overall probability $P_{h i g h}(j)$ of a run of $j$ large waves which after a little manipulation has the form:

$$
P_{\text {high }}(j)=p_{2,2}^{j-1}\left(1-p_{2,2}\right)
$$

\subsection{Quiescent Periods}

The present authors propose that the assumptions of the probability of a low runs underpinning a quiescent period require careful interpretation and are somewhat different to those employed by Kimura and Longeut Higgins (besides the obvious difference of seeking the low runs as opposed to the high runs). Clearly, a quiescent period of $j$ small wave heights is bounded at each end by large waves. Thus the authors considered it necessary to first incorporate the probability of the starting wave being large, i.e., $p_{2}$, and incorporate the conditional probability of a low wave occurring given that the large wave has occurred, i.e. $p_{2,1}$. The remainder of the argument equivalently follows the low runs equivalent to that adopted by Longuet-Higgins [15] for the high runs case. Thus, the probability $P_{Q P}(j)$ of a quiescent period of $j$ small waves bounded at each end by large waves has the form:

$$
P_{Q P}(j)=p_{2} p_{2,1} p_{1,1}^{j-1} p_{1,2}
$$

which is readily shown to have the form:

$$
P_{Q P}(j)=p_{1}\left(1-p_{1,1}\right)^{2} p_{1,1}^{j-1}
$$

An outcome of simply starting with a small wave is that this does not exclude the possibility that the precursor to this wave was similarly small and so on back in time. It is thus vital that the quiescent period starts with a large wave whose probability of occurrence must be incorporated.

\subsubsection{Cumulative Quiescence}

The practical quantity of interest to users wishing to exploit quiescent periods is typically not the probability of a given quiescent period of a specified length but the cumulative probability $P_{C Q P}(j)$ of all quiescent periods equal to and longer than $j$ waves, i.e.:

$$
P_{C Q P}(j)=\sum_{l=j}^{\infty} P_{Q P}(l)
$$

which, using the above evaluates to:

$$
P_{C Q P}(j)=p_{1}\left(1-p_{1,1}\right) p_{1,1}^{j-1}
$$


The probability $P_{C Q P}$ thus represents the probability of a quiescent period of at least $j$ small waves bounded at each end by a large wave.

\subsubsection{Quiescence Duration}

Further to the issue of user requirements, applications will mainly require the time duration of quiescence rather than the number of consecutive quiescent waves. The simplest approach which will be employed here, to this is to multiply the run length $j$ by an appropriate average period measure, such as the mean zero up-crossing period $\tau_{z}$.

If one wished to extend the present treatment a somewhat more sophisticated approach would be to compute a measure of the mean wave period, $\bar{\tau}\left(h<h_{c}\right)$, for wave heights over the restricted range $0<h<h_{c}$, then to multiply this by the run length $j$ corresponding to the particular $h_{c}$ value of interest. Given that the authors have employed the results from a band model in determining the runs length behaviour-it for consistency for this purpose it would be natural to use the a joint wave height $h$ period $\tau$ distribution $p(h, \tau)$ developed by from the same assumptions such as [20,21], i.e.:

$$
p(h, \tau)=L(v) \frac{2}{\pi^{0.5} v} \frac{\frac{h^{2}}{2 m_{0}}}{\left(\frac{\tau}{\tau_{1}}\right)^{2}} \exp \left(-\frac{h^{2}}{2 m_{0}}\left\{1+\frac{\left(1-\frac{\tau_{1}}{\tau}\right)^{2}}{v^{2}}\right\}\right)
$$

where the scaling constant, $L(v)$, is given by: $L(v)=2\left\{1+\left(1+v^{2}\right)^{-0.5}\right\}^{-1}$.

However as previously indicated it is the authors' view that for the purposes of a future simple low computational cost QPP operational planning tool, towards which this work is directed, it is deemed adequate to describe the quiescent periods in terms of a mean wave period measure and the number of individual small waves involved. Thus incorporating the above more sophisticated approach is left for our companion fully numerical approach publication.

\subsection{The Narrow Band Restriction and Quiescent Period Prediction:}

Both the envelope and the Markov chain approach use Rice's probability distribution $[13,14]$ (which are both linear narrow band approximations) strictly speaking require that the bandwidth parameter, $v<<1$, or at least $v$ is significantly less than unity. Several authors have addressed the issue of spectral bandwidth for wave systems with Cavanié et al [22] finding that even modest high frequency truncation of a spectrum such as Pierson Moskowitz had a very large effect on reducing $v$. This is especially relevant to the validity of narrow band theory to QPP applications because only the energy dominant 
section of the spectrum around the peak is typically important. Furthermore despite $v$ values for the cases examined being approximately 0.4 both Arhan and Ezraty [19] and Longuet-Higgins [23] found good agreement between wave statistics and metrics derived from linear narrow-band noise theory.

\section{Using Spectral Parameters for Wave-Wave Correlation}

\subsection{Correlation Coefficient between Successive Waves}

By employing either simulations or actual wave data a discrete version of the wave correlation coefficient $\gamma$ can be determined. As is well known $\gamma$ evaluated discretely in this manner is a biased estimator for the parameter $\kappa$ which is of interest here. The two quantities $\gamma$ and $\kappa$ were shown to be related to the by Uhlenbeck [24] and Middleton [25] as follows:

$$
\gamma=\frac{E(\kappa)-\frac{1}{2}\left(1-\kappa^{2}\right) K(\kappa)-\frac{\pi}{4}}{1-\frac{\pi}{4}}
$$

in which, $K$, and $E$, are complete elliptic integrals of the first and second kind respectively.

The inconvenience of having to use (12) to obtain $\kappa$ from $\gamma$ can be avoided by recognising that the parameter $\kappa$ can be obtained directly from the power density spectrum. This is achieved by using a discrete form of the Wiener-Khinchin theorem. Authors have discussed this approach $[15,18]$ from slightly different, but quantitatively equivalent, perspectives. Battjes [18] obtains $\kappa$ directly from the

magnitude of the normalised autocorrelation function $\frac{1}{m_{0}}|R(\tau)|$, derived from the wave spectrum evaluated at a lag value given by the mean zero crossing period, $\tau_{z}$. This exploits the fact that, $m_{0}=R(0)$

Thus:

$$
\kappa=\frac{1}{\int_{0}^{\infty} S(\omega) d \omega^{\infty}}\left|\int_{0}^{\infty} S(\omega) e^{i \omega \tau_{z}} d \omega\right|
$$


The present authors will follow Battjes [16] noting that: $\tau_{z}=2 \pi\left(\frac{m_{0}}{m_{2}}\right)^{\frac{1}{2}}$, the parameters, $m_{0}$ and $m_{2}$ being the zeroth and second moments of the energy spectrum respectively.

Longuet-Higgins [15] also derives, $\kappa$, via a normalised autocorrelation function which in this case is of the wave envelope. The lag value employed was, $\tau_{1}=2 \pi \frac{m_{0}}{m_{1}}$.

Specifically:

$$
\kappa=\frac{\sqrt{\left(X^{2}+Y^{2}\right)}}{m_{0}}
$$

where:

$$
X=\int_{0}^{\infty} S(\omega) \cos \left\{\left(\omega-\omega_{0}\right) \tau_{0}\right\} d \omega
$$

and:

$$
Y=\int_{0}^{\infty} S(\omega) \sin \left\{\left(\omega-\omega_{0}\right) \tau_{0}\right\} d \omega
$$

where, $\omega_{0}$, is the centre frequency of the narrow band spectrum which physically is interpreted as the angular frequency at which the envelope of wave heights is modulated.

Now provided, $\omega_{0}$, can be chosen such that, $\omega_{0}=\frac{2 \pi}{\tau_{0}}$, the approaches of Battjes and Longuet-Higgins become mathematically equivalent. In this regard an important practical point here is that for many commonly used wave spectral models the two mean wave period measures, $\tau_{1}$, and, $\tau_{z}$, are numerically very similar, e.g., for the Pierson Moskowitz spectrum, $\frac{\tau_{Z}}{\tau_{1}}=0.92$. So given that Rice's narrow band model $[13,14]$ only approximately applies to wave spectra to within the anticipated accuracy of the approach it is probably equally legitimate to use either value for the lag value $\tau_{0}$ at which to evaluate the normalised envelope auto-correlation function in the estimation of, $\kappa$. 


\subsection{Power Spectral Density Functions}

This inverse Fourier approach to estimating $\kappa$ is attractive because the statistical properties of sea waves are typically couched in terms of various classes of wave spectral model forms, e.g., Neumann, Pearson Moskowitz, Jonswap, etc. It is worth noting at this point that while it is common parlance in oceanographic research to either implicitly or explicitly refer to all wave spectral models as wave power spectra this is not formally correct. Apart from the obvious factor of 2 difference between these two quantities the main point is that the power density spectrum $S(\omega)$ is required to be an even function of $\omega$ whereas for many wave spectral models, including the Pierson Moskowitz spectrum, the wave spectra are odd functions of $\omega$.

It is the property of $S(\omega)$ being an even function that forces the use of the one-sided trigonometric integrals in Oceanographic work. This is necessary because the standard Fourier integral based definition of the power spectral density (ie., the Wiener-Khinchin theorem) spans $-\infty<\omega<\infty$ and clearly odd functions integrate to zero over this domain.

The strict correct analytic solution to this problem for situations of the present type where integrations are required over $-\infty<\omega<\infty$ is very simple and merely requires replacing the independent variable $\omega$ in spectral wave models exhibiting odd $\omega$ symmetry by the quantity $\frac{\omega}{2} S G N(\omega)$. The $S G N(\omega)$ simply returns the sign of $\omega$. In practice this is unnecessary as one can simply integrate over $0 \leq \omega<\infty$ and double the result as is implicitly done in oceanographic applications.

Hence one can say that when treated appropriately it is possible to employ the "one sided form" of the Wiener-Khinchin theorem to obtain $\kappa$ as given by:

$$
\kappa=\frac{1}{m_{0}}\left|R\left(\tau_{0}\right)\right|
$$

where given the assumption that $\omega_{0}=\frac{2 \pi}{\tau_{0}}$, the function, $R(\tau)$, evaluated at a lag of $\tau_{0}$ can be either the auto-correlation function of the wave elevations or of the wave envelope.

As stated the authors will follow Battjes [18] and employ, $\tau_{0}=\tau_{z}=2 \pi\left(\frac{m_{0}}{m_{2}}\right)^{\frac{1}{2}}$, corresponding to the mean zero-crossing wave period. Illustrative examples of $\tau_{z}$ are: for the Neumann spectrum, 
$\tau_{z}=\frac{U_{N}}{\sqrt{2 g}} \sqrt{\frac{3}{4}}$, and for the Pierson-Moskowitz form, $\tau_{z}=\frac{U_{P M}}{g \pi^{\frac{1}{4}}}$, where in each case the parameter $U$

is a wind speed value at a given height above sea level.

\section{A Significant General Property of Quiescence for a Range of Sea Types}

Consider the following spectral form:

$$
S(\omega)=\frac{B}{\omega^{\beta}} e^{-\frac{A}{\omega^{\alpha}}}
$$

This function encompasses the wave spectrum models of a wide range of sea states including the commonly used Neumann and Peirson-Moskowitz/Bretschnieder forms. In these models the constants, $B$ and $A$, are typically functions of wind speed over the region where the waves are formed but are independent of angular frequency $\omega$. This will also be assumed to be the true in the general case of (18). The only other restriction that will be placed upon (18) is that the parameters $\alpha$ and $\beta$ are real and positive.

Given (18) the moments $m_{0}$ and $m_{2}$ in terms of angular frequency (radian/sec), are given by:

$$
m_{0}=2 \int_{0}^{\infty} B \frac{e^{-\frac{A}{\omega^{\alpha}}}}{\omega^{\beta}} d \omega
$$

and:

$$
m_{2}=2 \int_{0}^{\infty} \omega^{2} B \frac{e^{-\frac{A}{\omega^{\alpha}}}}{\omega^{\beta}} d \omega
$$

Thus 


$$
\tau_{z}=2 \pi \sqrt{\frac{\int_{-\infty}^{\infty} \frac{e^{-\frac{1}{\eta^{\alpha}}}}{\eta^{\beta}} d \eta}{\int_{-\infty}^{\infty} \frac{e^{-\frac{1}{\eta^{\alpha}}}}{\eta^{\beta-2}} d \eta}} \frac{1}{A^{\frac{1}{\alpha}}}
$$

Using the change of variable: $\eta=\frac{\omega}{A^{\frac{1}{\alpha}}}$, the mean zero up-crossing wave period, $\tau_{z}$, can be written as:

Now recalling that:

$$
\left|R\left(\tau_{z}\right)\right|=\left|\int_{0}^{\infty} S(\omega) e^{i \omega \tau_{z}} d \omega\right| \frac{1}{m_{0}}
$$

and using the previous change of variables:

$$
\kappa=\left|\frac{1}{\int_{0}^{\infty} \frac{e^{-\frac{1}{\eta^{\alpha}}}}{\eta^{\beta}} d \eta} \int_{0}^{\infty} \frac{e^{-\frac{1}{\eta^{\alpha}}}}{\eta^{\beta}} \cos \left(\eta A^{\frac{1}{\alpha}} \tau_{z}\right) d \eta+j \frac{1}{\int_{0}^{\infty} \frac{e^{-\frac{1}{\eta^{\alpha}}}}{\eta^{\beta}} d \eta} \int_{0}^{\infty} \frac{e^{-\frac{1}{\eta^{\alpha}}}}{\eta^{\beta}} \sin \left(\eta A^{\frac{1}{\alpha}} \tau_{z}\right) d \eta\right|
$$

Substituting for $\tau_{z}$ gives: 


$$
\begin{gathered}
\kappa=\mid \frac{1}{\int_{0}^{\infty} \frac{e^{-\frac{1}{\eta^{\alpha}}}}{\eta^{\beta}} d \eta} \int_{0}^{\infty} \frac{e^{-\frac{1}{\eta^{\alpha}}}}{\eta^{\beta}} \cos \left(\eta 2 \pi \sqrt{\frac{\int_{0}^{\infty} \frac{e^{-\frac{1}{\eta^{\alpha}}}}{\eta^{\beta}} d \eta}{\int_{0}^{\infty} \frac{e^{-\frac{1}{\eta^{\alpha}}}}{\eta^{\beta-2}} d \eta}} d \eta\right. \\
+\sqrt{-1} \frac{1}{\int_{0}^{\infty} \frac{e^{-\frac{1}{\eta^{\alpha}}}}{\eta^{\beta}} d \eta} \int_{0}^{\infty} \frac{e^{-\frac{1}{\eta^{\alpha}}}}{\eta^{\beta}} \sin \left(\eta 2 \pi \sqrt{\frac{\int_{0}^{\infty} \frac{e^{-\frac{1}{\eta^{\alpha}}}}{\eta^{\beta}} d \eta}{\int_{0}^{\infty} \frac{e^{-\frac{1}{\eta^{\alpha}}}}{\eta^{\beta-2}} d \eta}}\right) d \eta \mid
\end{gathered}
$$

Equation (24) means that $\kappa$ is independent of the parameters $A$ and $B$ and hence independent of windspeed and thus of the significant wave height. Now the probability distribution $p\left(h_{2}, h_{1}\right)$, given by (2), only depends upon: (i) $\kappa$ and (ii) the wave heights normalised to the root-mean-square wave height. Furthermore the absolute dependences on wave height associated with the integrals over $p\left(h_{2}, h_{1}\right)$ and over the marginal probability density cancels because only the ratio of such integrals is used. Consequently, there is no dependence of the probability of a run of $j$ small waves on the absolute level of the prevailing wave height. Only the functional "form" of the spectrum through the parameters $\alpha$ and $\beta$ affects this.

Summarising, for the broad class of wave spectral object as defined by (18), the run of small waves only depends upon the "form" of the wave spectra and not upon the significant wave height. However, it must be born in mind that this finding only holds while the narrow band approximation holds legitimising the results of Rice. This analysis has been undertaken using $\tau_{z}=2 \pi \sqrt{m_{0} / m_{2}}$, however the same independence on absolute wave height can easily be shown by repeating the analysis if $\tau_{1}=2 \pi m_{0} / m_{1}$ is used rather than $\tau_{z}$.

Now as stated in section 3.3.2 it is the time duration of the "runs of small waves" that are most likely to interest users, rather than the number of waves, $j$, or rather the cumulative number of runs of at least this length. Hence as stated previously the simplest approach to this is for the number of consecutive small waves in a run to be multiplied by the corresponding mean wave period. Incorporating the wave period does introduce a dependence upon the absolute level of wave height, but using the average wave period approach this simply requires re-scaling the runs statistics and does not significantly add to the overall computational costs. 


\section{Semi-Analytic Evaluation of Quiescent Period Probabilities}

Given that the well-known parameterised spectral model forms are commonly employed for both describing and forecasting sea conditions, the ability to estimate the runs properties (in this case of QPs) of different categories of seas directly from such model spectra in the manner described can be of considerable value in developing future forward planning aids for maritime operations. This can be achieved for all power density spectra if the integrals involved in determining the low runs probability are evaluated numerically. As stated a companion work describes such a numerical approach and presents examples using power spectral densities obtained from both simulations and from actual sea trials data. However, for a set of interesting commonly met special cases encompassed by the form given in (18) (including the: Neumann, Peirson-Moskowitz and Bretschneider), somewhat surprisingly it also is possible to obtain semi-analytic results which significantly reduces computational costs and also demonstrates a further runs property that could reduce computational cost further.

Such a semi-Analytic approach will be developed here for the process described in sections 3 and 4 for spectra of the type: $S(\omega)=\frac{B}{\omega^{\beta}} e^{-\frac{A}{\omega^{\alpha}}}$. This will be illustrated for the special cases of the Neumann and the Pierson-Moskowitz/Bretschneider spectra. The early Neumann form is included because unlike the much more commonly cited Pierson-Moskowitz/Bretschneider spectra, it is an even function of the frequency and thus is a true power density function.

Given that the key parameter $\kappa$ is derived from auto-correlation functions, it is sensible to explore the literature on the analytic treatment of this quantity with respect to wave spectral models. Expressions for the autocorrelation functions for both Neumann and the Pierson-Moskowitz wave spectra have been obtained by Latta and Balie [26]. Their method for the Neumann case involved an exponential operator technique applied via the Mellin transform, and the Pierson-Moskowitz case was approached by setting up an associated differential equation. These methods were indirect, extremely intensive and very case specific. In contrast, the frequency domain approach employed here very readily yields analytic expressions for the respective envelope auto-correlations in terms of standard special functions. Specifically the key parameter $\kappa$ appearing in (2) can be evaluated analytically in terms of hypergeometric and MeijerG special functions, which are standard function calls in symbolic language packages (such as Maple and Mathematica). This means that one layer of numeric integration in the runs evaluation process can be avoided making it realistic to incorporate the present approach in computationally cheap user packages designed to provide advice on the quiescence conditions.

Given that the nomenclature of such special functions is rather cumbersome and these forms do not fall within most users mathematical experience, the functional details are omitted. Consequently it is simply stated here that for integer values of $\alpha$ and $\beta$ the required integrals of the type: 
$B \int_{0}^{\infty} \frac{e^{-\frac{A}{\omega^{\alpha}}}}{\omega^{\beta}} \cos \left(\omega \tau_{z}\right) \mathrm{d} \omega$

and,

$$
B \int_{0}^{\infty} \frac{e^{-\frac{A}{\omega^{\alpha}}}}{\omega^{\beta}} \sin \left(\omega \tau_{z}\right) \mathrm{d} \omega
$$

can be considered to be standard forms within the repertoire of typical symbolic language packages.

\subsection{Relevant Parameters of the Neumann, Pierson-Moskowitz/Bretschneider Spectra}

The Neumann wave spectral model $S_{N}(\omega)$ which is a true power density spectrum, is given by:

$$
S_{N}(\omega)=B_{N} \frac{e^{-\frac{A_{N}}{\omega^{2}}}}{\omega^{6}}
$$

where, $B_{N}=3.05 \frac{\pi}{2}$, and, $A_{N}=2 g^{2} U_{N}^{-2}$, in which, $g$, is the gravitational constant, and, $U_{N}$, the wind velocity $7.5 \mathrm{~m}$ above the sea surface. The first and second moments of this spectrum are given respectively by:

$m_{N, 0}=B_{N} 3 \sqrt{\frac{\pi}{2}}\left(\frac{U_{N}}{2 g}\right)^{5}$, and, $m_{N, 2}=B_{N} \sqrt{\frac{\pi}{2}}\left(\frac{U}{2 g}\right)^{3}$. The corresponding mean zero up-

crossing period is given by: $\tau_{z, N}=\frac{\sqrt{3}}{2} \frac{U}{g}$.

The Pierson Moskowitz form, which is not a true power spectral density function, is:

$$
S_{P M}(\omega)=B_{P M} \frac{e^{-\frac{A_{P M}}{\omega^{4}}}}{\omega^{5}}
$$


where, $B_{P M}=8.1 \times 10^{-3} g^{2}$, and, $A_{P M}=0.74\left(\frac{g}{U_{P M}}\right)^{4}$, where, $g$, is the gravitational constant and, $U_{P M}$, is the wind velocity $19.5 \mathrm{~m}$ above the sea surface. The two moments, $m_{0, P M}$, and, $m_{2, P M}$, are given by:

$m_{0, P M}=\frac{B_{P M}}{4 A_{P M}}$, and, $m_{2, P M}=B_{P M} \frac{\sqrt{\pi}}{4 \sqrt{A_{P M}}}$. The corresponding mean zero up-crossing period is

given by: $\tau_{z, \mathrm{PM}}=(0.74 \pi)^{1 / 4} \frac{U}{g}$.

From the perspective of QP analysis the Bretschneider form is mathematically equivalent to Pierson Moskowitz, the fact that the constants, $B_{B S}$, and, $A_{B S}$, are chosen independently does not affect the results. Thus the value of the wave-wave correlation parameter, $\kappa$, for these two forms is the same.

\section{Illustrations}

The analysis described in sections 2 to 5 is used in a comparison against results obtained from a linear sea wave simulation. The key issue is: what is the probability that under specific sea conditions of the occurrence of quiescent periods of at least $j$ consecutive wave of heights bounded above by $h_{c}$ ? Given the ubiquity of the Pierson Moskowitz/Bretschneider spectral forms this case was used as an illustration of the approach, employing a wind of speed $19 \mathrm{~m} \cdot \mathrm{sec}^{-1}$ at the standard reference height of $19.5 \mathrm{~m}$ above sea level. It should be recalled that the analytic results for such probabilities are independent of wind speed.

The simulations were generated in the standard manner by superimposing multiple sinusoids with phases sampled from a uniform distribution over $0 \rightarrow 2 \pi$, and magnitudes obtained by randomly sampling from a Rayleigh distribution whose variance is provided by $S(\omega)$. The latter process is especially important [27] in order to obtain simulations with the correct runs properties rather than being merely "sea like". In order to obtain confidence in the low probability events the simulations are run over an equivalent real time of 260,000 seconds using a sampling timestep of 1 second. The total number of zero up-crossings was approximately 28,000. The frequency band employed is $0.003 \mathrm{~Hz} \rightarrow 0.3 \mathrm{~Hz}$ and the deep water dispersion $k=\frac{\omega^{2}}{g}$ relationship is assumed. 
Fig. 1 compares the results of the present semi-analytic approach against the simulations. The parameter varied between each curve is the critical wave height value $h_{c}$. The results presented cover the range, $\frac{1}{6} \sqrt{2} h_{r} \leq h_{c} \leq \sqrt{2} h_{r} \leq h_{c}$.

The validity of the analytic prediction, based upon the narrow band model, that the runs lengths are independent of absolute wave height, is explored in the scatter diagram shown in figure 2 that uses data derived from two cases of wave height, i.e., $m_{0}$ values of $3.6 \mathrm{~m}$ and $6.7 \mathrm{~m}$. The results plotted are of the fractional change in the probability between the two cases. Given that the standard deviation of these changes is less than 10 percent (well within the anticipated precision of the methodology presented here) these results bear out the sea state independence for the Pierson Moskowitz form and it seems not unreasonable to assume that the same is likely to be true for the whole class of spectral forms given by equation 26 . 


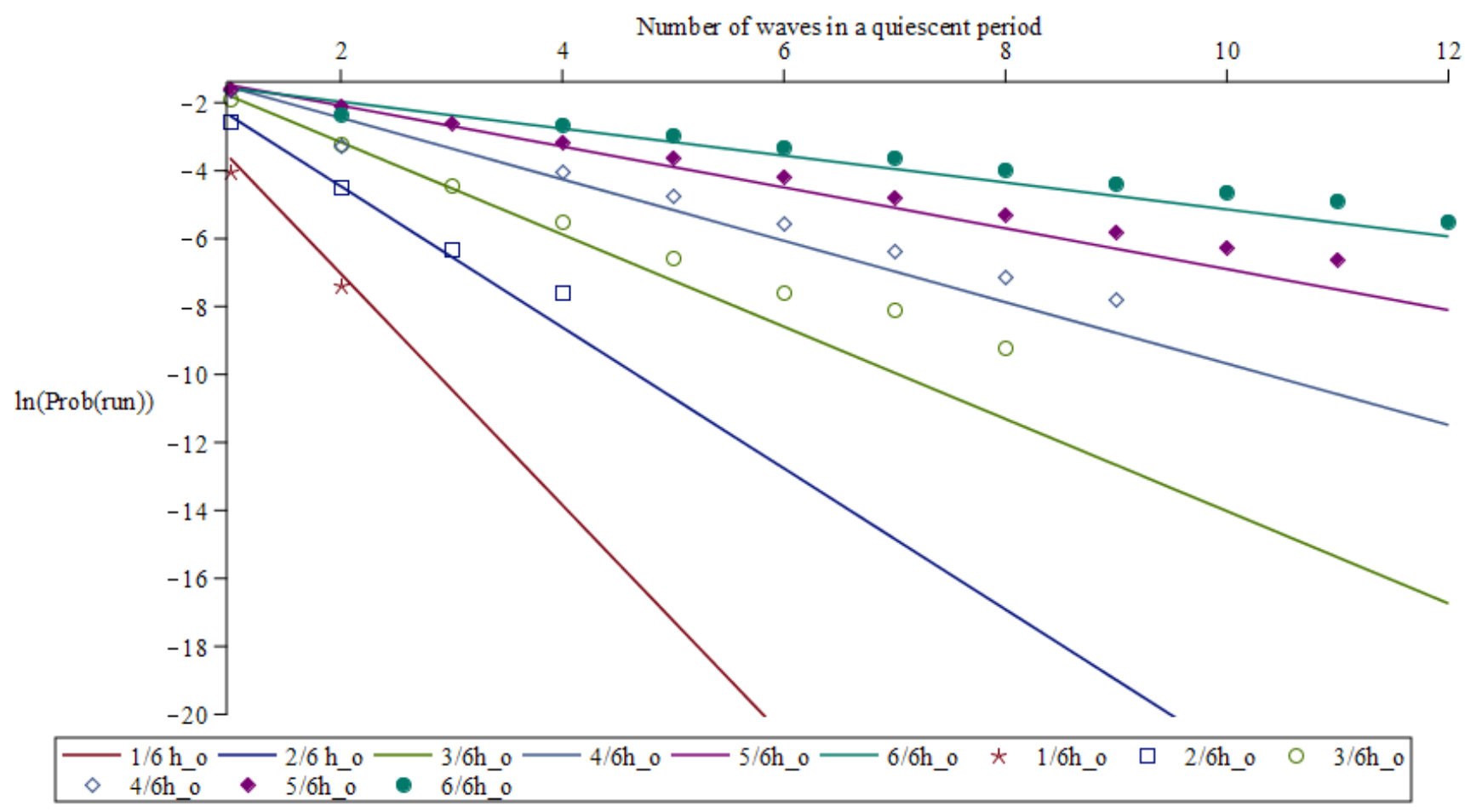

Figure 1. Plots of the natural logarithm of the probability of a given number of runs within a quiescent period. The parameter, h_o, is equal to $\sqrt{2} h_{r}$, where, $h_{r}$, is the root mean square sea surface elevation. The data derived from the wave simulations described is denoted by discrete symbols while the predictions correspond to solid lines. 


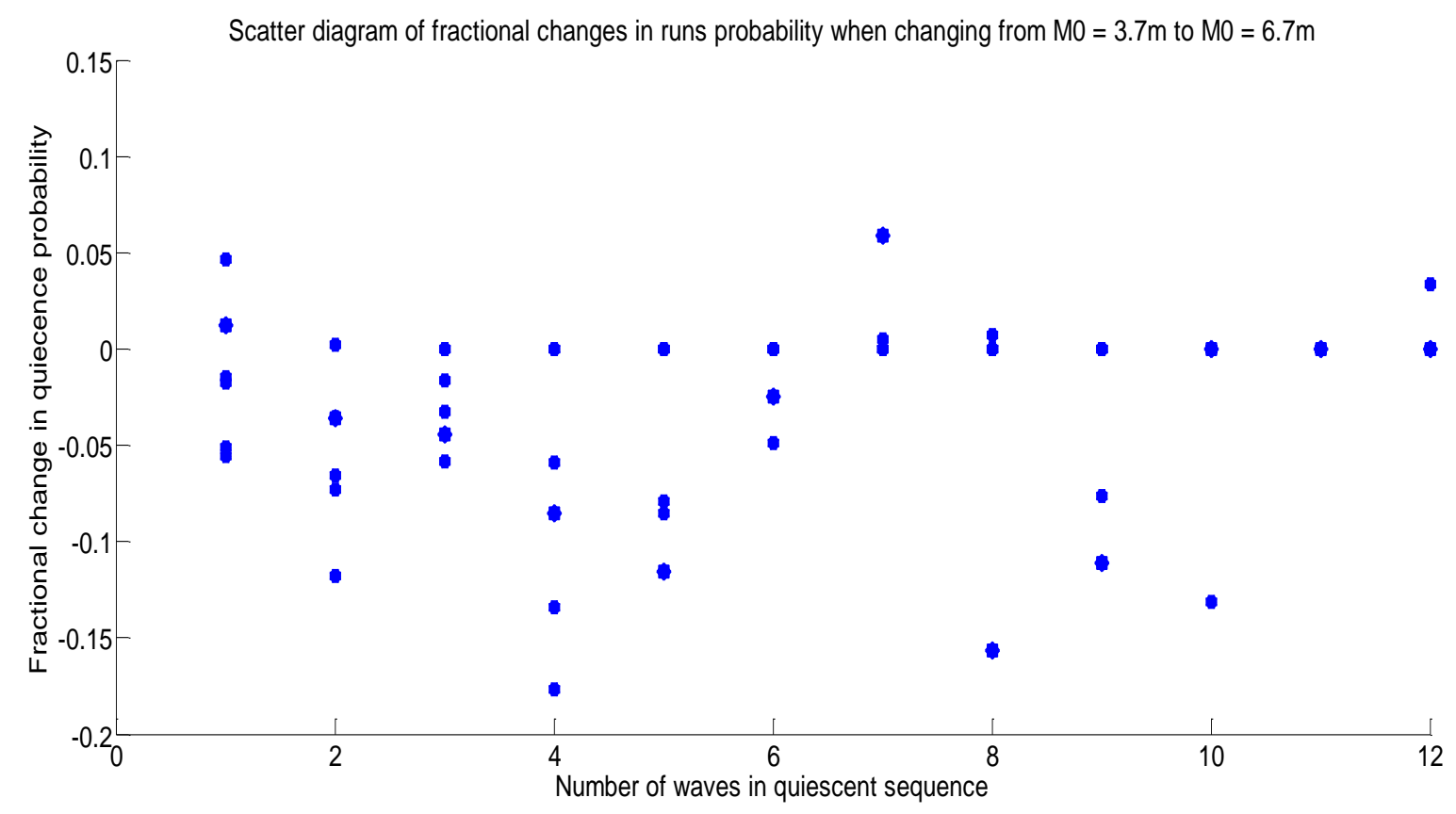

Figure 2. A scatter diagram derived from the simulations of the fractional change in the probability of quiescence when the wind velocity increases from $19 \mathrm{~m} / \mathrm{sec}$ to $22 \mathrm{~m} / \mathrm{sec}$, corresponding to $m_{0}$ rising from $3.7 \mathrm{~m}$ to $6.7 \mathrm{~m}$. The range of critical wave heights over which the data is determined is equivalent to that in figure 1.

\section{Discussion and Conclusions}

The results presented in figure 1 and figure 2 show a reasonable agreement between the simulations and the semi-analytic calculations using the present approach and demonstrate that under conditions where QPP might be employed the runs statistics are at most only weakly sensitive to absolute sea state. Because of the rarity of these events, the fits between theory and the simulations become increasingly worse as the number of waves present in the quiescent period increases. The type of conditions where deterministic QPP might expect to be used (aircraft or small boat recovery and cargo transfers) would be typified by large well developed swell seas produced by remote storms with a dominant wave period of 10 to $12 \mathrm{sec}$. Typical prediction times of 1 minute are considered to be operationally useful in such applications [1]-[3] which in the above context is approximately 5 wave periods. To set this in context the results in 
figure 1 indicated that quiescence probability of 5 wave periods can be reasonably well estimated provided the critical wave heights, $h_{c}$, is given by, $h_{c} \geq \frac{3}{6} \sqrt{2} h_{r}$, i.e., $h_{c} \geq \frac{h_{r}}{\sqrt{2}}$.

The purpose of the present work is to estimate the statistics of quiescence. This methodology is designed to be used to estimate if there is likely to be sufficient quiescence present for the deterministic estimation of such quiescence during the operation to be of value. So in assessing the potential value of the proposed techniques the real comparison that must be made is to compare using deterministic methods in an application against to simply employing traditional sea state measures. If an activity is deemed to be safe below wave height $h_{c}$ and the mean square value under the prevailing conditions is $h_{r}$ then using traditional statistics one would need to decide a confidence level $\varepsilon\left(h_{c}\right)$ which it is deemed to be sufficiently low i.e., obtaining $h_{c}$ is sufficiently unlikely so as to be acceptably small. This would inevitably mean that $h_{c}$ would have to be a very small fraction of the prevailing root mean square value $h_{r}$. At the very least $h_{c} \geq 4 h_{r}$ . In contrast with Deterministic Sea Wave Prediction the occurrence of the actual expected wave height values, rather than their probabilities can be estimated. The critical criterion then is the accuracy of DSWP which given the $h_{c} \geq 4 h_{r}$ alternative condition for the statistical approach means that DSWP could actually be very imprecise and still be useful.

Thus deterministic QPP would be of considerable operational benefit and the present semianalytic approach would provide a useful tool to aid in the development of a future QPP forward planning tool.

Of considerable practical importance is the fact that theoretical estimates tend to underestimate the probability of quiescence, the converse being clearly operationally dangerous.

Hence the following specific conclusions can be drawn:

1. For wave spectra investigated the cumulative probability of a quiescent period of at least $j$ small waves predicted by the analysis is a reasonable fit to simulations and is independent of absolute wave height. Thus figure 1 can be taken to be a generic plot for the Pierson Moskowiz/Bretschneider case as a whole, for all wind speeds and hence all wave heights.

2. The standard bandwidth width measure, $v=\left(\frac{m_{0, P M} m_{2, P M}}{m_{1}^{2}}-1\right)^{\frac{1}{2}}$, is also wind speed independent [28]. Thus at least for Moskowitz/Bretschneider spectra the level of error 
introduced in using Rice's narrow band theory is also wind speed/wave height independent.

3. Conversion to the duration of quiescence does introduce a dependence on the prevailing wind speed, $U$, and to a first approximation is simply a matter of multiplying the $j$ values in figure 1 by $\tau_{z, \mathrm{PM}}=(0.74 \pi)^{1 / 4} \frac{U}{g}$.

\section{Acknowledgments}

This work was supported by the EPSRC Research Grant EP/N009142/1.

\section{REFERENCES}

1. J. M. Giron-Sierra, S. Esteban. The Problem of Quiscent Period prediction for Ships: A Review. $8^{\text {th }}$ IFAC Conference on Control Applications in Marine Systems. Rostock-Warnmünde, Germany Sept 15-17, 2010. PP307-311.

2. J. Duncan et al (2012). The Development of the Quiescent Period Prediction System by Simulation. Proceedings of the ASNE Launch and Recovery Symposium. Arlington.

3. Belmont, J Christmas, B Ferrier, JM Duncan and J Duncan. UK. Progress in Quiescent Period Prediction. Warship 2016: Advanced Technologies in Naval Design, Construction, \& Operation, 15-16 June 2016, Bath, UK.

4. E.L. Morris, H.K. Zienkiewicz, M.M.A. Purzanjani, J.O. Flower and M.R. Belmont. Techniques for Sea State Prediction. 2nd. Int. Conf. On Manoeuvring and Control of Marine Craft, Southampton, pp 547-571, July 1992.

5. M.R. Belmont_and J. Christmas, J. Dannenberg, J. Duncan and J.M. Duncan and B. Ferrier. An Examination of the Feasibility of Linear Deterministic Sea Wave Prediction in MultiDirectional Seas Using Wave Profiling Radar: Theory, Simulation and Sea Trials. Journal of Atmospheric and Oceanic Technology, Vol. 31, No. 7, pp1601-1614, 2014.

6. T.Hilmer, and E. Thornhill, 2014: Deterministic wave predictions from the WaMoS II, Proc.Of OCEANS'14, Taipei, 1-8. 
7. T.Hilmer, and E.Thornhill, 2015 :Observations of Predictive Skill for Real-Time Deterministic Sea Waves from the WaMoS II. Proc. of OCEANS'15, Washington, 1-7.

8. E. Blondel, G. Ducrozet, F. Bonnefoy and P. Ferrant. Deterministic Reconstruction and prediction of No-linear Wave Systems. Intl. Workshop on Water Waves and Floating Bodies. 2008.

9. Y. Goda. Numerical experiments on wave statistics with spectral simulation. Rept. Port and Harbour Res. Inst., Vol .9, No. 3, pp.3-75, 1970.

10. J.R. Wilson, and W. F. Baird : A discussion of some measured wave data, Proc. 13th Conf. Coastal Engineering, 1972, pp.113-130.

11. H. Rye. Wave group formation among storm waves, Proc. 14th Conf.Coastal Engineering, 1974, pp.164-183.

12. W. Siefert. Wave investigation in shallow water, Proc. 14th Conf. Coastal Engineering, 1974, pp.151-178.

13. S.O.Rice. The mathematical analysis of random noise. Bell Syst. Tech. J. 23, 282-332: 24, 46-156, (1944-1945).

14. S.O.Rice. Distribution of the duration of fades in radio transmission Gaussian noise model. Bell Syst. Tech. J. 37, 581-635, (1958).

15. M. S. Longuet-Higgins. "Statistical Properties of Wave Groups in a Random Sea State". Philosophical Transactions of the Royal Society of London. Series A, Mathematical and Physical Sciences, Vol. 312, No. 1521, (Oct. 12, 1984), pp. 219-250.

16. B. Boashash, "Estimating and Interpreting the Instantaneous Frequency of a Signal-Part I: Fundamentals", Proceedings of the IEEE, Vol. 80, No. 4, pp. 519-538, April 1992.

17. A. Kimura. Statistical properties of random sea groups. Proc. $17^{\text {th }}$ Int. Conf. Coastal 18. J.A. Battjes and G.Ph. van Vledder. Verification of Kimura's theory for wave group statistics. Proc. 19 ${ }^{\text {th }}$ Int. Conf. Coastal Engrg. (Houston ASCE) (1984) pp 642-648.

19. M. Arhan and R. Ezraty. Statistical relations between successive wave heights. Oceanologica Acta, 1978, Vol. 1, No. 2, pp15-22. 
20.M.S. Longuet-Higgins, M. S. 1952, On the statistical distribution of the heights of sea waves, J. Mar. Res., 11, 245-266.

21. M.S. Longuet-Higgins, 1980, On the distribution of heights of sea waves: some effects of nonlinearity and finite band width, J Geophys Res 85: 1519-1523.

22. A. Cavanié, M.Arhan and R. Ezraty. A statistical relationship between individual heights and periods of storm waves. BOSS 76, Trondheim, pp 235-244, 1976.

23. M.S. Longuet-Higgins. Proc. R. Soc. Lond. A 389, pp 241-258, 1983.

24. G.E.Uhlenbeck. Theory of random processes. M.I.T. radiation Lab. Rep. 454, October 1943.

25. Middleton. Statistical Communication theory. New York: McGraw Hill, 1960.

26. G.E.Latta and J.E.Balie. On the Autocorrelation Functions of Wind Generated Ocean Waves. Zeitschrift Angewandte Mathematic und Physik, Vol. 19, pp 575-586, 1969.

27. M.J.Tucker, P. G. Challenor, and D. J. T. Carter, 1984: Numerical simulation of a random sea: a common error and its effect upon wave group statistics. Appl. Ocean Res., 6, 118-122.

28. M.J. Tucker and E.G. Pitt. Waves in ocean Engineering. Vol. 5 - $1^{\text {st }}$ Edit. Elsevier, 2001.. 